\title{
A special issue in honor of Irene Pepperberg: The chemist of comparative cognition
}

\author{
Debbie M. Kelly ${ }^{1}$ Lauren M. Guillette ${ }^{2}$ \\ Accepted: 20 January 2021 / Published online: 11 February 2021 \\ (C) The Psychonomic Society, Inc. 2021
}

We are honored to edit this special issue of Learning \& Behavior to celebrate Irene Pepperberg's remarkable scientific career. The Comparative Cognition Society selected Dr. Irene Pepperberg to receive the Society's annual Research Award for 2020. This recognition is bestowed upon an individual who has been selected by their scientific peers to have made an "outstanding contribution to the study of comparative cognition in animals." As the recipient of this award, a symposium of invited colleagues and students was organized to highlight the scientific influence of Irene's research over the years. Irene was invited to deliver a Master Lecture to conclude the symposium. Although these events were scheduled to occur at the 27th International Conference on Comparative Cognition (CO3), the conference was canceled as the world grappled with the consequences of COVID-19. Instead, these events will now be held in the Spring of 2021, giving us another year to celebrate Irene's achievements. This current Special Issue of Learning \& Behavior, inspired through the organization of the symposium, is a tribute highlighting the broad and substantial impact Irene's research has had on the field of comparative cognition.

Irene Pepperberg is most famously known for her research into the cognitive and communicative abilities of animals. Most notably her studies with the language-trained grey parrot Alex, and more recently Griffin and Athena, have revolutionized how the scientific and public communities think about avian cognition. The field of comparative cognition is truly fortunate to have attracted the inquisitive and analytical mind of Irene Pepperberg; as some may not know, her Ph.D. is in Chemical Physics from Harvard University. After completing

Debbie M. Kelly

Debbie.Kelly@umanitoba.ca

1 Department of Psychology, University of Manitoba, 190 Dysart Road, Winnipeg, MB R3T 2N2, Canada

2 Department of Psychology, University of Alberta, Edmonton, AB, Canada her postdoctoral fellowship in animal-human communication at Purdue University in 1979, Irene accepted a position as Research Associate in the Department of Biology at Purdue. During 1984, Irene moved to Northwestern University as a Visiting Assistant Professor in the Department of Anthropology until 1991, when she accepted a position as Associate Professor at the University of Arizona in the Department of Ecology and Evolutionary Biology, with a joint appointment in the Department of Psychology. During 1999, Irene took on the position of Visiting Associate Professor at Massachusetts Institute of Technology Media Lab, where over the following years she accepted the position of Research Scientist. Irene was recruited to Brandeis University in 2002 as a Research Associate Professor and later an Adjunct Associate Professor. During this time, Irene was a Radcliffe Institute Fellow and Lecturer for the Department of Psychology at Harvard University, where she is currently a Research Associate.

Irene's dedication to and perseverance with investigating cognition and communication helped forge a path for our understanding of the cognitive capabilities of animals, particularly with respect to symbolic representation, numerical competence, and inferential reasoning. Her books Alex \& $M e$, which was a New York Times best-seller and won a Christopher Award, and The Alex Studies: Cognitive and Communicative Abilities of Grey Parrots, exemplify Irene's commitment to making scientific research, and the importance of animal cognition, assessable to the general public. These volumes, alongside her numerous empirical articles, detail the intensive training program Irene established to tap into the cognitive abilities of grey parrots. Indeed, a memory that comes readily to mind is talking to Irene about comparative cognition, while a grey parrot perches inquisitively on her shoulder. The dedication Irene has for research parallels that of her strong commitment to supporting colleagues and the mentorship of students. The latter was most recently acknowledged by the John R. Marquand Award for Excellence in Advising and Support to Harvard undergraduates. 
This special edition of Learning \& Behavior, focusing primarily on avian cognition, reflects the breadth of influence that Irene's scientific knowledge has had on the field of comparative cognition. Irene's approach to understanding animal cognition has been interdisciplinary in nature. Thus, this special issue includes articles from many views, including cognitive biology, an approach Irene has supported enthusiastically over her career. This perspective is represented by a review of convergent evolution of complex cognition from the lens of self-awareness by Baciadonna and colleagues. Highlighting the importance of considering the ecological environment, Vernouillet and colleagues examine whether the presence of conspecifics influence caching decisions by pinyon jays, and Schwing and colleagues show that when dominant Kea learn restraint, cooperation may be facilitated.

Irene's research has had a substantial impact on the field's inquiries and knowledge of the numerical competencies of animals. This contribution is reflected by articles examining whether birds discriminate quantity. Lemaire and colleagues approached this topic by showing that male and female domestic chicks respond differently to changing numerousness using imprinting procedures in the laboratory, whereas using a foraging paradigm with wild, free-ranging birds, Corliss and colleagues show that rufous humming birds have an approximate number system.

Understanding the cognitive processes involved in discrimination and problem solving have been prevailing areas of interest throughout Irene's research program. Highlighting the former, a paper by Wright and colleagues compares same-different discrimination and the effect of proactive interference among three avian and two primate species. The influence of Irene's work can also be seen in the elegant tests using novel flexible open-ended choice discriminations examining depth perception in complex scenes by pigeons in a laboratory task by Gray and colleagues. Zentall takes a comparative approach examining the ephemeral reward task across different species. A paper by O'Neill and colleagues examines problem-solving abilities in two species of nonhabitually tool-using macaw parrots, and Wakonig and colleagues examine how Goffin's cockatoos consider causeeffect relationships during string pulling tasks. The commonalities in these papers not only demonstrate the influence of Irene, but they also take a truly comparative approach to understanding cognitive abilities in animals.

Given Irene's infamous research using the model-rival with grey parrots, this edition would not be complete without studies of communication. A paper by Beecher and Akçay review work on song sparrows to ask if song tutoring may qualify as true teaching by paying attention to different aspects of social factors: direct interaction of tutor with tutee or via social eavesdropping. A paper by Osmanski and colleagues examines, using operant conditioning in the laboratory, the role of location and quality (visual vs. auditory) cues in vocal production learning in budgerigars. Gallego-Abenza head back to the field to determine if for ravens, vigilance may be influenced not only by who is calling out the presence of a predator, but when.

We hope you enjoy the variety of topics presented in this special issue. We feel the broad range of topics and the international representation of colleagues and students truly reflect the influence Irene has had, and continues to have, on the field of comparative cognition.

Publisher's note Springer Nature remains neutral with regard to jurisdictional claims in published maps and institutional affiliations. 